\title{
Alterations in plasma B-type natriuretic peptide levels after repair of congenital heart defects: A potential perioperative marker
}

Chie-Youn Shih, MD, ${ }^{a}$ Anil Sapru, MD, ${ }^{\text {a }}$ Peter Oishi, MD, ${ }^{a}$ Anthony Azakie, MD, ${ }^{\mathrm{b}}$ Tom R. Karl, MD, ${ }^{\mathrm{b}}$ Cynthia Harmon, BS, Ritu Asija, MD, ${ }^{a}$ Ian Adatia, MB, ChB, ${ }^{a}$ and Jeffrey R. Fineman, MD ${ }^{\mathrm{a}, \mathrm{c}}$

Objectives: B-type natriuretic peptide, a cardiac hormone with diuretic, natriuretic, and vasoactive properties, is used in the diagnosis, risk stratification, and management of adult cardiac patients. However, no study has yet determined the prognostic value of B-type natriuretic peptide after surgical intervention for congenital heart disease. The objectives of this study were (1) to determine alterations in B-type natriuretic peptide levels after repair of congenital heart disease with cardiopulmonary bypass and (2) to investigate potential associations between B-type natriuretic peptide levels and outcomes in this patient population.

Methods: Fifty-one infants and children undergoing repair of congenital heart disease were studied. B-type natriuretic peptide levels were measured before and after surgical intervention, and the ability of the postoperative 12-hour B-type natriuretic peptide level to predict postoperative outcomes was evaluated.

Results: B-type natriuretic peptide levels increased after separation from cardiopulmonary bypass, with an 8 -fold peak increase at 12 hours $(P<.005)$. Postoperative 12-hour B-type natriuretic peptide levels were associated with the duration of mechanical ventilation and the presence of a low cardiac output state after surgical intervention. On multivariate analysis, the 12-hour B-type natriuretic peptide level was an independent predictor of the duration of mechanical ventilation. In fact, B-type natriuretic peptide levels of greater than $540 \mathrm{pg} / \mathrm{mL}$ predicted mechanical ventilation beyond 48 hours, with a sensitivity of $88.9 \%$ and a specificity of $82.5 \%$. In addition, B-type natriuretic peptide levels of greater than $815 \mathrm{pg} / \mathrm{mL}$ predicted the presence of a low cardiac output state within 48 hours after surgical intervention, with a sensitivity of $87.5 \%$ and a specificity of $90.2 \%$.

From the Department of Pediatrics, ${ }^{a}$ Department of Surgery, ${ }^{\mathrm{b}}$ and the Cardiovascular Research Institute, ${ }^{\mathrm{c}}$ University of California, San Francisco, Calif.

This research was supported in part by grants HL61284 and MO1RR01271 (JRF) from the National Institutes of Health.

Received for publication Sept 23, 2005; revisions received Oct 26, 2005; accepted for publication Oct 31, 2005.

Address for reprints: Jeffrey R. Fineman, MD, Department of Pediatrics, 505 Parnassus Ave, Box 0106, San Francisco, CA 94143 (E-mail: jeff.fineman@ucsf.edu).

J Thorac Cardiovasc Surg 2006;131:632-8

$0022-5223 / \$ 32.00$

Copyright $\odot 2006$ by The American Association for Thoracic Surgery

doi:10.1016/j.jtcvs.2005.10.052

Conclusions: B-type natriuretic peptide determinations might be a useful tool for clinicians caring for infants and children after surgical intervention for congenital heart disease.

$\mathrm{D}$ espite numerous advances, surgical intervention for patients with congenital heart disease continues to carry significant morbidity and mortality. Accordingly, considerable resources, including specialized intensive care, are used postoperatively, and yet up to $25 \%$ of infants and young children have diminished cardiac output within the first 24 hours. ${ }^{1,2}$ Management during this period is complicated by a dearth of validated physiologic markers to guide therapy, resulting in a substantial reliance on subjective clinical assessments. Direct measurements of hemodynamic indices, such as cardiac output, are often not possible in these patients because of altered blood flow (eg, intracardiac shunting), size limitations that preclude invasive monitoring, or both, making the continued search for such reliable markers important. 


\section{Abbreviations and Acronyms \\ $\mathrm{BNP}=\mathrm{B}$-type natriuretic peptide \\ $\mathrm{CHF}=$ congestive heart failure \\ $\mathrm{CPB}=$ cardiopulmonary bypass \\ PCICU $=$ Pediatric Cardiac Intensive Care Unit \\ $\mathrm{SvO}_{2}=$ mixed venous oxyhemoglobin saturation}

B-type natriuretic peptide (BNP) is a 32-amino-acid polypeptide hormone with diuretic, natriuretic, and vasoactive properties that is secreted by the cardiac ventricles in response to myocyte stretch. ${ }^{3}$ Determinations of BNP are increasingly used in the diagnosis, risk stratification, and management of adult cardiac patients. ${ }^{4-7}$ Much less data exist on the role of BNP in pediatric patients with cardiac disease, and no study has yet determined the prognostic value of BNP levels after surgical repair of structural congenital heart defects. ${ }^{8-13}$

The objectives of this study were thus (1) to determine alterations in BNP levels over time after repair of congenital heart defects with cardiopulmonary bypass (CPB) and (2) to investigate potential associations between BNP levels and outcomes in this patient population. We prospectively studied 51 infants and children undergoing complete repair of structural congenital heart defects with CPB. Systemic arterial plasma BNP determinations were made before and 2, 12, and 24 hours after CPB. We then evaluated the ability of the 12-hour BNP level to predict postoperative outcomes.

\section{Methods \\ Subjects}

This prospective cohort study was conducted at the Pediatric Cardiac Intensive Care Unit (PCICU) at the University of California, San Francisco. Patients with congenital heart defects undergoing complete surgical repair with $\mathrm{CPB}$ were enrolled. Patients were excluded from the study if they required staged singleventricle palliation or were unable to separate from CPB and required extracorporeal life support postoperatively.

The patients were followed up during their entire course in the PCICU. The perioperative anesthesia management, CPB strategy, and subsequent PCICU management followed standard institutional practices. After surgical repair, all patients were admitted to the PCICU intubated and mechanically ventilated. An on-service team that was blinded to the BNP values made all decisions regarding patient management.

Written informed consent was obtained from the patients' parents or guardians before enrollment of the patients into the study. The institutional review board at the University of California, San Francisco, reviewed and approved this study.

\section{Data Collection}

Blood samples were obtained from an arterial catheter preoperatively and at 2, 12, and 24 hours after CPB. The samples were immediately placed on ice in prechilled ethylenediamine tetraace- tic acid tubes and centrifuged at $3000 \mathrm{rpm}$ for 15 minutes at $4^{\circ} \mathrm{C}$. Separated plasma was stored at $-20^{\circ} \mathrm{C}$. Within 4 days of obtaining the sample, the plasma was thawed to room temperature, and BNP levels were measured with a commercially available fluorescence immunoassay (Triage Meter Plus, Biosite Diagnostic). The measurable range of BNP on this device is between 5 and $5000 \mathrm{pg} / \mathrm{mL}$. The average $95 \%$ confidence limit of the analytic sensitivity for the Triage BNP test is less than $5 \mathrm{pg} / \mathrm{mL}$.

Clinical and laboratory data were prospectively collected at each sampling point and once daily thereafter by an observer blinded to the BNP data. The clinical data collected included demographics, CPB time, crossclamp time, duration of mechanical ventilation, inotrope dosage, mean systemic arterial pressure, central venous pressure, and fluid balance. Laboratory data included base deficit, venous blood gases, and serum lactate levels.

\section{Preoperative Classification of Cardiac Defect}

Cardiac lesions were classified as having decreased, normal, or increased pulmonary blood flow to investigate associations between preoperative BNP levels and preoperative cardiac physiology. Lesions classified as having decreased pulmonary blood flow included pulmonary atresia, tetralogy of Fallot, and pulmonary stenosis. Lesions classified as having increased pulmonary blood flow included atrial septal defect, ventricular septal defect, atrioventricular septal defect, truncus arteriosus, transposition of the great arteries, and totally anomalous pulmonary venous return.

\section{Calculations}

Inotrope use was quantified by a score adapted from Wernovsky and colleagues. ${ }^{2}$ The score was calculated by obtaining the total amount of inotropic support the patients received at each sampling point and then entering the data into the following equation:

Dopamine + Dobutamine $+([$ Epinephrine

$$
+ \text { Norepinephrine }] \times 100)=\text { Milrinone } \times 20) .
$$

Units of inotrope dosage used in this equation were in micrograms per kilogram per minute.

The definition of a low cardiac output state was identical to criteria published by Hoffman and coworkers. ${ }^{1}$ Low cardiac output state was defined by a combination of changes in clinical signs and biochemical indicators and the administration of interventions aimed at augmenting cardiac output, including increased pharmacologic support relative to the baseline and mechanical pacing. Clinical signs included tachycardia, oliguria, poor perfusion, or cardiac arrest occurring with or without a widened arterial-mixed venous oxyhemoglobin saturation $\left(\mathrm{SvO}_{2}\right)$ difference or metabolic acidosis.

\section{Analysis of the Data}

Analysis of continuous variables within categories was made with $t$ tests and analysis of variance or the nonparametric MannWhitney and Kruskal-Wallis tests, as appropriate. Because of their nonnormal distribution, BNP levels were log transformed. A linear regression model was used to assess the association between the log-transformed 12-hour BNP levels and the primary predictor (ie, the duration of mechanical ventilation). A logistic regression model was used to assess the association between 12-hour BNP 
TABLE 1. Demographics and patient characteristics

\begin{tabular}{lc}
\hline Demographics & $331(1-5655)$ \\
Age (d), median (range) & $9.6(2.5-76)$ \\
Weight (kg), median (range) & $55 \%$ \\
Male sex & $45 \%$ \\
Female sex & $n$ \\
Type of lesion & 9 \\
ASD & 4 \\
AV canal & 2 \\
Aortic insufficiency & 3 \\
Aortic stenosis & 1 \\
Mitral insufficiency & 1 \\
Mitral stenosis & 1 \\
Pulmonary atresia & 2 \\
Pulmonary insufficiency & 1 \\
TAPVR & 7 \\
Tetralogy of Fallot & 5 \\
Transposition of the great arteries & 4 \\
Truncus arteriosis & 9 \\
VSD & $81(25-157)$ \\
CPB time (min), median (range) & $44(0-87)$ \\
Crossclamp time (min), median (range) &
\end{tabular}

$A S D$, Atrial septal defect; $A V$, atrioventricular; TAPVR, total anomalous pulmonary venous return; $V S D$, ventricular septal defect; $C P B$, cardiopulmonary bypass.

levels and the presence of a low cardiac output state. Next, the duration of mechanical ventilation was dichotomized to being equal to or less than 48 hours or greater than 48 hours. Receiver operating characteristics curves were used to assess the various cutoff values of BNP to predict (1) the need for mechanical ventilation beyond 48 hours and (2) the development of a low cardiac output state within 48 hours of surgical intervention.

\section{Results}

Fifty-one patients were initially enrolled in this study. However, 2 patients could not be separated from CPB, and they required extracorporeal life support and were therefore excluded from the study. Of the 49 patients included in the study, 27 were male and 22 were female. Their ages ranged from 1 day to 15 years of age. The types of cardiac defects, demographics, and other baseline data are shown in Table 1. There was no mortality among the enrolled patients during the study period.

Preoperative BNP levels ranged from 5 to $1140 \mathrm{pg} / \mathrm{mL}$. There was an inverse relationship between preoperative BNP levels and weight $\left(\mathrm{rho}_{\mathrm{s}}=-0.51, P<.002\right)$ and age $\left(\mathrm{rho}_{\mathrm{s}}=-0.48, P<.0006\right)$. There was no difference in the levels of preoperative BNP with the sex of the patient $(P=$ .19). Although there was a trend toward increasing BNP levels across categories of increasing pulmonary blood flow, this difference was not statistically significant. Twenty study patients were treated for congestive heart failure (CHF) before surgical intervention. There was a statistically significant difference in preoperative BNP levels between
TABLE 2. BNP levels before and after CPB

\begin{tabular}{lrrcr}
\hline Time points & $\mathbf{N}$ & $\begin{array}{r}\text { Median } \\
(\mathbf{p g} / \mathbf{m L})\end{array}$ & Interquartile range & $\boldsymbol{P}$ value \\
\hline Before CPB & 48 & 38.3 & $13.3-96.8$ & $<.0001$ \\
2 h after CPB & 47 & 58.3 & $22.2-363.0$ & \\
$12 \mathrm{~h}$ after CPB & 49 & 301.0 & $166.0-642.0$ & \\
$24 \mathrm{~h}$ after CPB & 48 & 261.0 & $150.0-413.0$ &
\end{tabular}

$B N P$, B-type natriuretic peptide; $C P B$, cardiopulmonary bypass.

patients treated for $\mathrm{CHF}$ and those not treated for $\mathrm{CHF}$ (median values, 82.6 vs $14.7 \mathrm{pg} / \mathrm{mL} ; P<.006$ ).

On serial follow-up, BNP levels increased over time after discontinuation of $\mathrm{CPB}$, reaching a peak at 12 hours $(P<.001$, Table 2$)$. Postoperatively, BNP levels reached a peak at 12 hours in $62 \%$ of the patients. The change in BNP over time is depicted in Figure 1. The 12-hour BNP level had a significant correlation with the duration of mechanical ventilation (rho $=0.66, P<.0001$ ), bypass time (rho $=0.51, P<.0002$ ), and aortic crossclamp time (rho $=0.28, P<.046$ ). The relationship between the log-transformed hours of mechanical ventilation and BNP levels was linear (Figure 2). On univariate linear regression analysis, the 12-hour BNP level was a significant predictor of the duration of mechanical ventilation $\left(r^{2}=0.32\right.$, coefficient $=13.5, P<.0001)$. Other variables that were predictors of the duration of mechanical ventilation on univariate analysis included preoperative BNP level, bypass time, 12-hour mean systemic arterial pressure, and age. Variables that were not associated with the duration of mechanical ventilation included 12-hour inotrope score, 12 hour lactate level, 12-hour central venous pressure, and

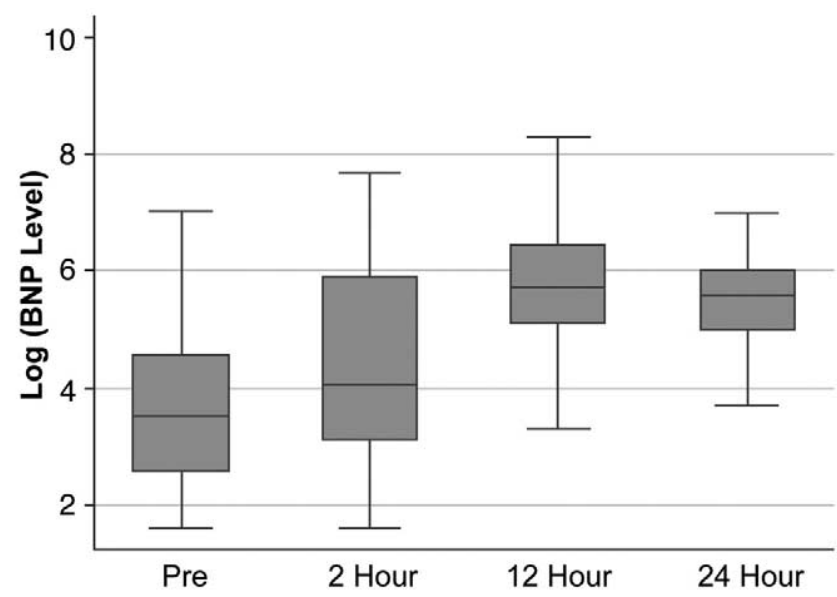

Figure 1. Box plots showing the change in B-type natriuretic peptide (BNP) before and after cardiopulmonary bypass. Boxes show the interquartile range, and $I$-bars represent the highest and lowest values. BNP levels are log transformed because of nonnormal distribution. 


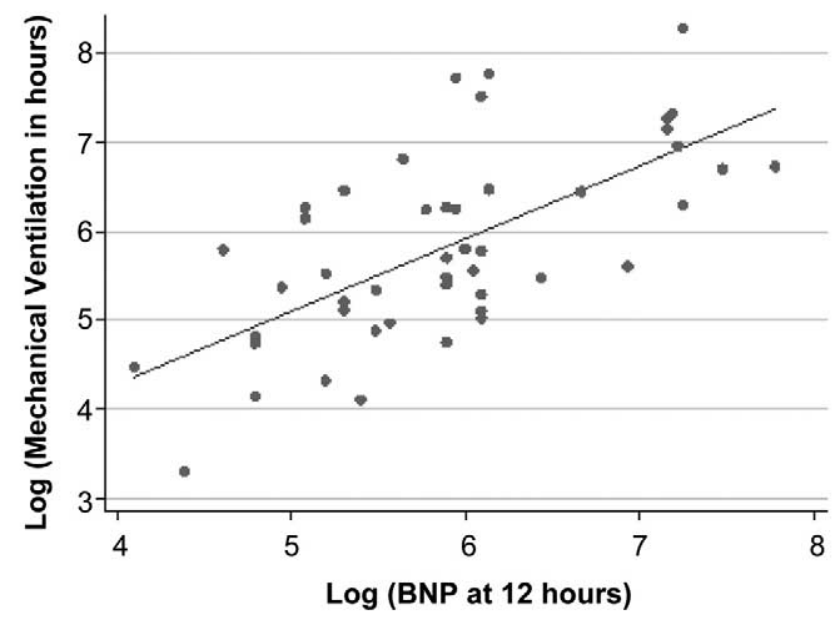

Figure 2. Scatter plot showing the relationship between 12-hour B-type natriuretic peptide (BNP) levels and the duration of mechanical ventilation. Because of nonnormal distribution, the data have been log transformed.

24-hour fluid balance. On multivariate linear regression analysis, by a backward stepwise elimination model, the 12-hour BNP level was an independent predictor of the duration of mechanical ventilation.

Twelve-hour BNP levels were higher in the 15 patients requiring mechanical ventilation beyond 48 hours $(P<$ .0002 , Figure 3 ). A receiver operating curve, which was used to evaluate the various cutoff levels of 12-hour BNP to

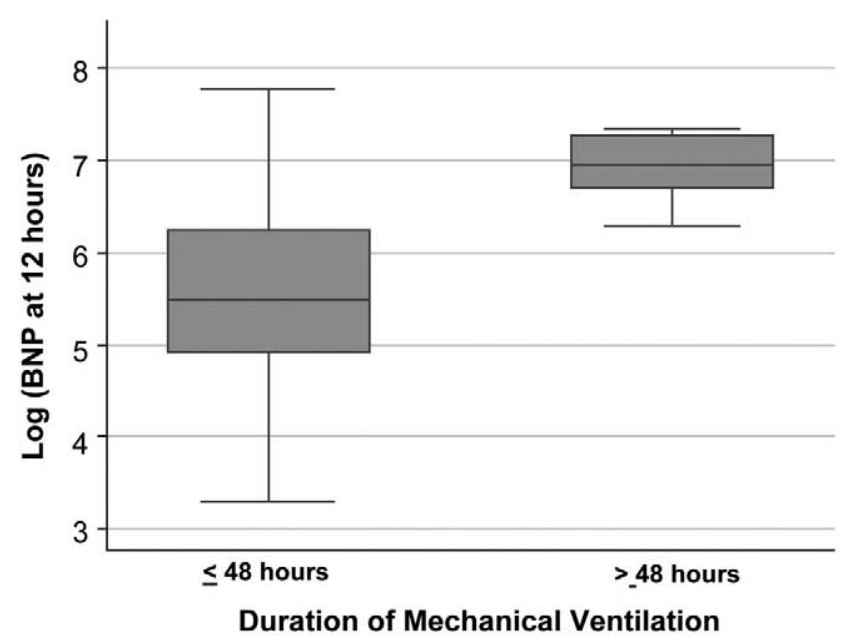

Figure 3. Box plots showing the relationship of 12-hour B-type natriuretic peptide (BNP) to the need for mechanical ventilation beyond 48 hours. Boxes show the interquartile range, and I-bars represent the highest and lowest values. Twelve-hour BNP levels are log transformed because of nonnormal distribution.

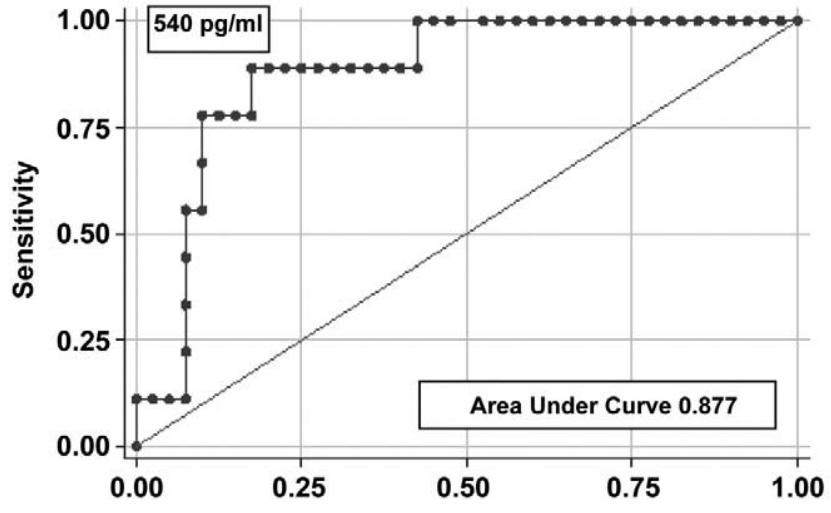

Figure 4. Receiver operating characteristic curve of the various cutoff levels of 12-hour B-type natriuretic peptide in predicting the need for mechanical ventilation beyond 48 hours. A cutoff value of $540 \mathrm{pg} / \mathrm{mL}$ has a sensitivity of $88.9 \%$ and a specificity of $82.5 \%$.

predict the need for mechanical ventilation beyond 48 hours after surgical intervention, had an area under the curve of $87.7 \%$ (95\% confidence interval, 76.8\%-96.8\%; Figure 4). A 12-hour BNP cutoff value of $540 \mathrm{pg} / \mathrm{mL}$ or more had a sensitivity of $88.9 \%$, a specificity of $82.5 \%$, a positive predictive value of $54 \%$, and a negative predictive value of $97 \%$ for predicting the need for mechanical ventilation beyond 48 hours. No patient in this study required reintubation.

Twelve-hour BNP levels were also higher in the 11 patients who had a low cardiac output state within the first 48 hours after CPB $(P<.0001$, Figure 5). On logistic regression, development of a low cardiac output state within the first 48 hours had an odds ratio of 1.28 for every $100-p g$ increase in the 12-hour BNP level. A receiver operating curve, which was used to evaluate the various cutoff levels of 12-hour BNP to predict the development of a low cardiac output state within 48 hours after CPB, had an area under the curve of $91.4 \%$ (95\% confidence interval, $80 \%-100 \%$; Figure 6). A 12-hour BNP cutoff level of $815 \mathrm{pg} / \mathrm{mL}$ or more had a sensitivity of $87.5 \%$, a specificity of $90.2 \%$, a positive predictive value of $64 \%$, and a negative predictive value of $97 \%$ for predicting the presence of a low cardiac output state within the first 48 hours.

\section{Discussion}

The principal findings of this study are as follows: (1) BNP levels increase in pediatric patients with congenital heart defects after surgical repair with CPB and (2) BNP levels 12 hours after CPB are predictive of a requirement for mechanical ventilation beyond 48 hours and the presence of a low cardiac output state within the first 48 hours postoperatively. This is the largest study to date evaluating perioperative BNP levels in infants and children undergoing com- 


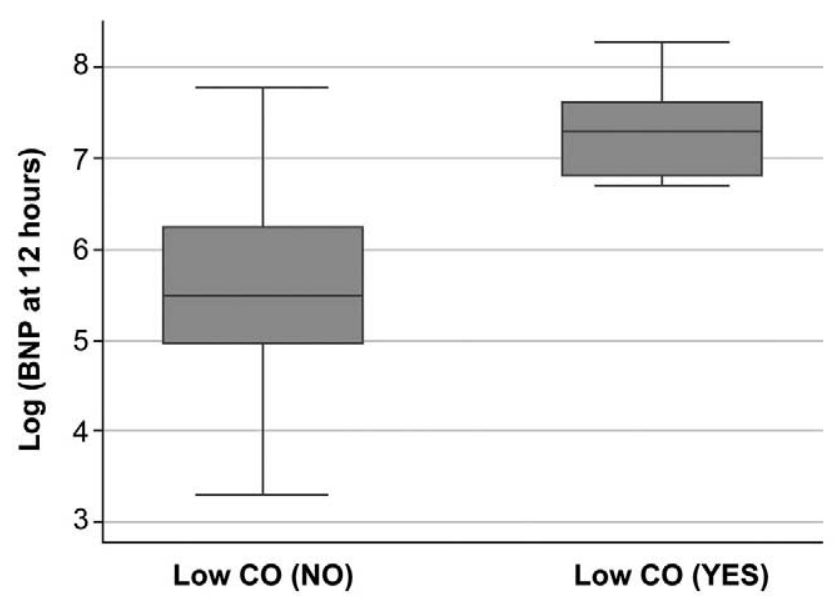

Figure 5. Box plots showing the relationship of 12-hour B-type natriuretic peptide (BNP) levels to the development of a low cardiac output within 48 hours after cardiopulmonary bypass. Boxes show the interquartile range, and I-bars represent the highest and lowest values. Twelve-hour BNP levels are log transformed because of nonnormal distribution.

plete repair of congenital heart defects. Moreover, to our knowledge, this is the first study to demonstrate the prognostic utility of BNP levels in this population, suggesting an important role for their use in postoperative management.

BNP is a member of a structurally related group of peptide hormones, termed natriuretic peptides, that also includes atrial natriuretic peptide and C-type natriuretic peptide. BNP is produced predominantly in the cardiac ventricles. The primary actions of BNP are vascular smooth muscle relaxation, diuresis, and natriuresis. ${ }^{3,14}$ Of the natriuretic peptides, BNP has emerged as the most useful marker for the severity of adult cardiac disease. ${ }^{4,5,15}$ We hypothesized that BNP would be a physiologically relevant marker of cardiac dysfunction in infants and children after surgical intervention for congenital heart disease.

Three studies have previously reported on changes in BNP after CPB in a pediatric population..$^{9,13,16}$ Most recently, Sun and colleagues ${ }^{13}$ measured BNP levels before and after surgical intervention in 27 patients undergoing biventricular repair and 27 patients undergoing univentricular repair of congenital heart defects. Plasma BNP levels increased after bypass in patients with biventricular defects but not in patients with univentricular defects, a group of patients not included in our study. Ationu and associates ${ }^{16}$ measured perioperative BNP levels in 9 children undergoing repair of congenital heart defects. Contrary to our findings, BNP levels decreased at 12 hours after surgical intervention, which might relate to the inclusion of 4 patients who underwent total cavopulmonary connection. ${ }^{16}$ Finally, Costello and coworkers ${ }^{9}$ examined BNP levels before and

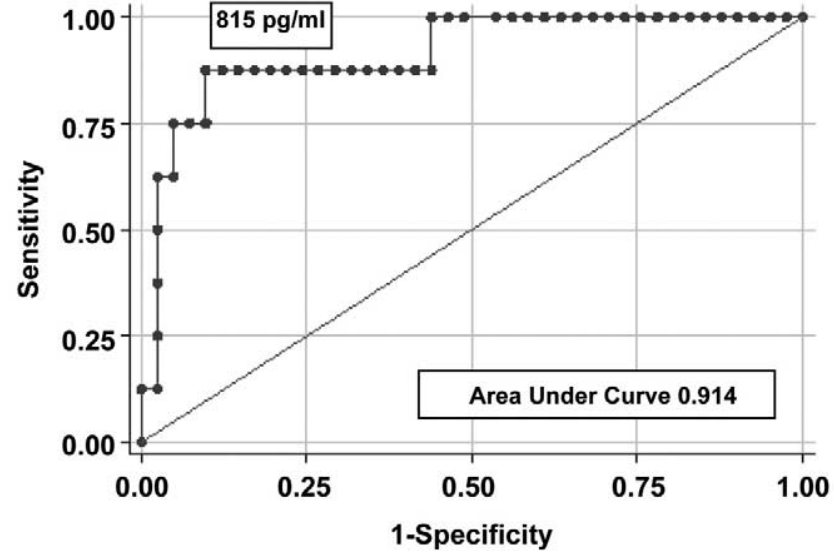

Figure 6. Receiver operating characteristic curve of the various cutoff levels of 12-hour B-type natriuretic peptide in predicting the development of a low cardiac output state within 48 hours after surgical intervention. A cutoff value of $815 \mathrm{pg} / \mathrm{mL}$ has a sensitivity of $\mathbf{8 7 . 5 \%}$ and a specificity of $\mathbf{9 0 . 2} \%$.

after cardiac surgery with $\mathrm{CPB}$ in 5 infants with $\mathrm{CHF}$ caused by left-to-right intracardiac shunts. Consistent with our data, BNP levels increased from preoperative levels at the first postoperative day. These studies are limited by their sample size and do not address correlations between BNP and postoperative clinical outcomes.

These issues have been better explored in the adult population. A number of investigators have described a consistent increase in BNP levels after cardiac surgery with CPB ${ }^{17-19}$ Furthermore, BNP levels have been associated with the presence and severity of ventricular dysfunction after surgical intervention. ${ }^{20}$ Finally, BNP levels have been associated with the extent of cardiac support required postoperatively, the duration of postoperative intensive care, and the incidence of cardiac events up to 2 years after surgical intervention. ${ }^{18-20}$ Our study is in keeping with these findings but is unique in the demonstrated association between postoperative BNP levels and the duration of mechanical ventilation and in the evaluation of pediatric patients.

To our knowledge, this is the first report that describes an association between BNP levels and the duration of mechanical ventilation after cardiac surgery. The duration of mechanical ventilation is commonly interpreted as an important marker of disease severity. Indeed, at our institution, most pediatric patients do not require mechanical ventilation beyond the first 24 to 36 hours after cardiac surgery. Therefore, we believed that dichotomizing the duration of mechanical ventilation to less than or greater than 48 hours would provide a clinically relevant outcome measure. In fact, there was significant overlap between patients requiring mechanical ventilation beyond 48 hours and those who 
had a low cardiac output state within 48 hours postoperatively. Validated criteria for extubation do not exist for infants and children after repair of congenital heart disease. Therefore, the attending physician made decisions regarding extubation on an individual basis. These physicians were blinded to the BNP values, and decisions regarding the timing of extubation conformed to the general approach of the cardiac intensive care team, which includes extubating within the first 24 hours when possible.

We report that BNP levels 12 hours after separation from CPB predict the development of a low cardiac output state within the first 48 hours postoperatively. Eleven (22\%) patients had a low cardiac output state within this period. This incidence is similar to that reported by several other investigators who determined cardiac output in pediatric patients after surgical intervention for congenital heart disease. ${ }^{1,2}$ Interestingly, BNP levels peaked at 12 hours after $\mathrm{CPB}$, which correlates with the onset of a low cardiac output state in these studies as well. ${ }^{1,2} \mathrm{We}$ did not use direct measurements of cardiac output (eg, dye dilution) in our definition of a low cardiac output state but rather used a composite definition that incorporates clinical signs, biochemical data, and the use of inotropic agents, vasoactive agents, or both, or other interventions (eg, pacing) directed at augmenting cardiac output. Although direct measures of cardiac output would have been valuable, they were not consistently available in our population. Importantly, physicians blinded to the BNP data made all therapeutic decisions, including those that influenced the definition of a low cardiac output state. Furthermore, the incidence of a low cardiac output state in our study was similar to that reported in studies that did directly measure cardiac output. ${ }^{2,21}$

Although the peak BNP level occurred 12 hours after bypass and most strongly predicted the need for prolonged mechanical ventilation, it is noteworthy that 6 of the 11 patients who had low cardiac output did so within the first 12 hours. Therefore, these patients had low cardiac output either before or at the time of the 12-hour BNP sampling, preventing the prognostic utility of this level. However, both the preoperative BNP level (odds ratio, 2.1 per 100-pg increase in BNP; $P=.012$ ) and the 2-hour BNP level (odds ratio, 1.2 per 100 -pg increase in $\mathrm{BNP} ; P=.013$ ) were also predictive of the development of low cardiac output. Therefore, these earlier time points might also have clinical utility in this patient population.

We found that preoperative BNP levels were higher in patients undergoing medical treatment for CHF. This is consistent with a number of studies that have established an association between BNP levels and the severity of ventricular dysfunction in patients with congenital heart defects. ${ }^{22-26}$ Furthermore, we found an inverse relationship between preoperative BNP levels and patient age and weight. Developmental studies indicate that BNP levels are highest at birth but decrease by the first week of life and by 2 weeks of age are generally lower than adult levels. ${ }^{27}$ Despite higher BNP levels in small infants, who might be expected to require a prolonged duration of mechanical ventilation, BNP levels were independently predictive of the duration of mechanical ventilation on multivariate regression analysis.

Our data indicate that 12-hour postoperative BNP levels might be useful in the management of patients with congenital heart defects. Because clinical assessments have limitations, biochemical tests that might help guide therapy in the postoperative period are important. A number of studies have documented a significant correlation between serum lactate levels and various outcome measures. ${ }^{28,29}$ Increased serum lactate levels reflect inadequate tissue oxygen delivery with resultant anaerobic metabolism and thus confirm an inadequate cardiac output, as opposed to heralding one. BNP release is stimulated by increased ventricular volume or pressure, and thus levels could potentially increase in advance of a decrease in tissue oxygen delivery. $\mathrm{SvO}_{2}$ is increasingly used as an indirect measure of cardiac output in pediatric patients after cardiac surgery. ${ }^{30} \mathrm{SvO}_{2}$ changes rapidly with changes in cardiac output and thus enables the clinician to titrate therapy in advance of tissue hypoxia. Unfortunately, accurate measures of $\mathrm{SvO}_{2}$ are often not possible because of vascular access limitations, intracardiac shunting, or both, which can diminish the reliability of the measured values.

Several limitations of the present study are noteworthy. First, we excluded patients undergoing palliation of singleventricle cardiac defects. Although we believed that our initial analysis would be best focused on a homogeneous population (ie, infants and children undergoing complete repair of congenital heart defects), the exclusion of this population prevents us from determining the utility of BNP levels in the management of our most tenuous patients. Future studies are planned to include these patients. Indeed, the stimuli for BNP release (ie, ventricular volume and pressure) might make it a particularly useful clinical marker for patients with single-ventricle disease, especially in regard to the timing of a cavopulmonary connection. In addition, this initial investigation contained a heterogeneous population of study patients with varying risks of perioperative morbidity and mortality. Future studies on more homogenous populations are warranted. Last, patients requiring extracorporeal life support were excluded from our analysis. BNP levels were measured at time points after $\mathrm{CPB}$ that were determined a priori. Thus, we could not include patients who were unable to be separated from $\mathrm{CPB}$ postoperatively.

Myocardial dysfunction after surgical intervention for congenital cardiac disease is a common and complex problem. Alterations in levels of BNP, a cardiac hormone, reflect 
aberrant myocardial dynamics in a manner not captured by other physiologic markers. Indeed, our data indicate that BNP might be a better predictor of prolonged ventilation than other more commonly used measures, such as bypass time, mean systemic arterial pressure, lactate levels, and fluid balance. Further studies are warranted to investigate the utility of BNP as a therapeutic guide in this population.

\section{References}

1. Hoffman TM, Wernovsky G, Atz AM, Kulik TJ, Nelson DP, Chang $\mathrm{AC}$, et al. Efficacy and safety of milrinone in preventing low cardiac output syndrome in infants and children after corrective surgery for congenital heart disease. Circulation. 2003;107:996-1002.

2. Wernovsky G, Wypij D, Jonas RA, Mayer JE Jr, Hanley FL, Hickey PR, et al. Postoperative course and hemodynamic profile after the arterial switch operation in neonates and infants. A comparison of low-flow cardiopulmonary bypass and circulatory arrest. Circulation. 1995;92:2226-35.

3. Yandle TG. Biochemistry of natriuretic peptides. J Intern Med. 1994; 235:561-76.

4. Doust JA, Glasziou PP, Pietrzak E, Dobson AJ. A systematic review of the diagnostic accuracy of natriuretic peptides for heart failure. Arch Intern Med. 2004;164:1978-84.

5. Doust JA, Pietrzak E, Dobson A, Glasziou P. How well does B-type natriuretic peptide predict death and cardiac events in patients with heart failure: systematic review. BMJ. 2005;330:625.

6. Lubien E, DeMaria A, Krishnaswamy P, Clopton P, Koon J, Kazanegra $\mathrm{R}$, et al. Utility of B-natriuretic peptide in detecting diastolic dysfunction: comparison with Doppler velocity recordings. Circulation. 2002;105:595-601.

7. Troughton RW, Frampton CM, Yandle TG, Espiner EA, Nicholls MG, Richards AM. Treatment of heart failure guided by plasma aminoterminal brain natriuretic peptide (N-BNP) concentrations. Lancet. 2000; 355:1126-30

8. Cowley CG, Bradley JD, Shaddy RE. B-type natriuretic peptide levels in congenital heart disease. Pediatr Cardiol. 2004;25:336-40.

9. Costello JM, Backer CL, Checchia PA, Mavroudis C, Seipelt RG, Goodman DM. Alterations in the natriuretic hormone system related to cardiopulmonary bypass in infants with congestive heart failure. Pediatr Cardiol. 2004;25:347-53.

10. Ationu A, Carter ND. Brain and atrial natriuretic peptide plasma concentrations in normal healthy children. Br J Biomed Sci. 1993;50: 92-5.

11. Kawamura T, Wago M. Brain natriuretic peptide can be a useful biochemical marker for myocarditis in patients with Kawasaki disease. Cardiol Young. 2002;12:153-8.

12. Holmstrom H, Omland T. Natriuretic peptides as markers of patent ductus arteriosus in preterm infants. Clin Sci (Lond). 2002;103:79-80.

13. Sun LS, Dominguez C, Mallavaram NA, Quaegebeur JM. Dysfunction of atrial and B-type natriuretic peptides in congenital univentricular defects. J Thorac Cardiovasc Surg. 2005;129:1104-10.

14. Levin ER, Gardner DG, Samson WK. Natriuretic peptides. $N$ Engl J Med. 1998;339:321-8.
15. Cowie MR, Struthers AD, Wood DA, Coats AJ, Thompson SG, Poole-Wilson PA, et al. Value of natriuretic peptides in assessment of patients with possible new heart failure in primary care. Lancet. 1997;350:1349-53.

16. Ationu A, Singer DR, Smith A, Elliott M, Burch M, Carter ND. Studies of cardiopulmonary bypass in children: implications for the regulation of brain natriuretic peptide. Cardiovasc Res. 1993;27:1538-41.

17. Avidan MS, Meehan N, Ponte J, El-Gamel A, Sherwood RA. Changes in brain natriuretic peptide concentrations following open cardiac surgery with cardioplegic cardiac arrest. Clin Chim Acta. 2001;303: 127-32.

18. Watanabe M, Egi K, Hasegawa S, Tanaka H, Ohshima H, Sakamoto T, et al. Significance of serum atrial and brain natriuretic peptide release after coronary artery bypass grafting. Surg Today. 2003;33:671-3.

19. Cuthbertson BH, McKeown A, Croal BL, Mutch WJ, Hillis GS. Utility of B-type natriuretic peptide in predicting the level of peri- and postoperative cardiovascular support required after coronary artery bypass grafting. Crit Care Med. 2005;33:437-42.

20. Morimoto K, Mori T, Ishiguro S, Matsuda N, Hara Y, Kuroda H. Perioperative changes in plasma brain natriuretic peptide concentrations in patients undergoing cardiac surgery. Surg Today. 1998;28: 23-9.

21. Parr GV, Blackstone EH, Kirklin JW. Cardiac performance and mortality early after intracardiac surgery in infants and young children. Circulation. 1975;51:867-74.

22. Nir A, Bar-Oz B, Perles Z, Brooks R, Korach A, Rein AJ. N-terminal pro-B-type natriuretic peptide: reference plasma levels from birth to adolescence. Elevated levels at birth and in infants and children with heart diseases. Acta Paediatr. 2004;93:603-7.

23. Mir TS, Marohn S, Laer S, Eiselt M, Grollmus O, Weil J. Plasma concentrations of $\mathrm{N}$-terminal pro-brain natriuretic peptide in control children from the neonatal to adolescent period and in children with congestive heart failure. Pediatrics. 2002;110:e76.

24. Westerlind A, Wahlander H, Lindstedt G, Lundberg PA, Holmgren D. Clinical signs of heart failure are associated with increased levels of natriuretic peptide types B and A in children with congenital heart defects or cardiomyopathy. Acta Paediatr. 2004;93:340-5.

25. Law YM, Keller BB, Feingold BM, Boyle GJ. Usefulness of plasma B-type natriuretic peptide to identify ventricular dysfunction in pediatric and adult patients with congenital heart disease. Am J Cardiol. 2005;95:474-8.

26. Kunii Y, Kamada M, Ohtsuki S, Araki T, Kataoka K, Kageyama M, et al. Plasma brain natriuretic peptide and the evaluation of volume overload in infants and children with congenital heart disease. Acta Med Okayama. 2003;57:191-7.

27. Koch A, Singer H. Normal values of B type natriuretic peptide in infants, children, and adolescents. Heart. 2003;89:875-8.

28. Munoz R, Laussen PC, Palacio G, Zienko L, Piercey G, Wessel DL. Changes in whole blood lactate levels during cardiopulmonary bypass for surgery for congenital cardiac disease: an early indicator of morbidity and mortality. J Thorac Cardiovasc Surg. 2000;119:155-62.

29. Charpie JR, Dekeon MK, Goldberg CS, Mosca RS, Bove EL, Kulik TJ. Serial blood lactate measurements predict early outcome after neonatal repair or palliation for complex congenital heart disease. J Thorac Cardiovasc Surg. 2000;120:73-80.

30. Tweddell JS, Hoffman GM. Postoperative management in patients with complex congenital heart disease. Semin Thorac Cardiovasc Surg Pediatr Card Surg Annu. 2002;5:187-205. 